\title{
Fulminant arterial vasculitis as an unusual complication of disseminated staphylococcal disease due to the emerging CC1 methicillin-susceptible Staphylococcus aureus clone: a case report
}

\author{
Charles Vidal ${ }^{1}$, Florence Moulin ${ }^{2}$, Xavier Nassif ${ }^{1}$, Louise Galmiche ${ }^{3}$, Delphine Borgel ${ }^{4}$, Alain Charbit ${ }^{5}$, \\ Capucine Picard ${ }^{6,7}$, Jean-Paul Mira ${ }^{8,9}$, Olivier Lortholary ${ }^{10}$, Anne Jamet ${ }^{1,5}$ and Julie Toubiana ${ }^{9,11^{*}}$ (D)
}

\begin{abstract}
Background: Staphylococcus aureus has emerged as a leading cause of invasive severe diseases with a high rate of morbidity and mortality worldwide. The wide spectrum of clinical manifestations and outcome observed in staphylococcal illness may be a consequence of both microbial factors and variability of the host immune response.

Case presentation: A 14-years old child developed limb ischemia with gangrene following S. aureus bloodstream infection. Histopathology revealed medium-sized arterial vasculitis. The causing strain belonged to the emerging clone CC1-MSSA and numerous pathogenesis-related genes were identified. Patient's genotyping revealed functional variants associated with severe infections. A combination of virulence and host factors might explain this unique severe form of staphylococcal disease.
\end{abstract}

Conclusion: A combination of virulence and genetic host factors might explain this unique severe form of staphylococcal disease.

Keywords: S. aureus, Sepsis, Vasculitis, Virulence, Genetic susceptibility, Polymorphism, Case report

\section{Background}

Staphylococcus aureus is a major human pathogen and a global healthcare issue. Humans are a natural reservoir of $S$. aureus, which can occasionally cause diseases that range in severity from minor skin infections to severe cases of pneumonia, bacteremia and septic shock [1]. The severity and outcome of the infection relies on bacterial virulence, as $S$. aureus is known to have a wide arsenal of components that contribute to the pathogenesis of infection. S. aureus is the cause of septic shock through cell wall components eliciting production of

\footnotetext{
* Correspondence: julie.toubiana@aphp.fr

${ }^{9}$ Department of Infection, Immunity and Inflammation, Institut Cochin, INSERM U1016, Paris, EU, France

${ }^{11}$ Department of General Pediatrics and Pediatric Infectious Diseases, Necker Enfants-Malades Hospital, APHP, Paris Descartes University, 149 rue de Sèvres, 75015 Paris, EU, France

Full list of author information is available at the end of the article
}

inflammatory cytokines through TLR2 pathway activation in innate immune cells [2]. S. aureus also contains several toxins that are able to potentiate hostinflammatory response, target and injure leukocytes and tissues, and inhibit bacterial clearance [3]. Finally, S. aureus avidly adheres to endothelial cells and platelet-fibrin thrombi involved in the physiopathology of endocarditis or thrombophlebitis [4]. Recently, the role of genetic factors of the host has been extensively studied, revealing their influence on the susceptibility to or the severity of sepsis. Rare monogenic inborn errors of immunity were found to predispose to severe infectious diseases, such as mutations that impair NF- $\mathrm{KB}$ responses [5]. In parallel, common variants of genes involved in innate immune response, inflammation and coagulation during sepsis were found to be associated with severity of bacterial infections $[6,7]$. We report here a severe and atypical phenotype of infection

(c) The Author(s). 2019 Open Access This article is distributed under the terms of the Creative Commons Attribution 4.0 International License (http://creativecommons.org/licenses/by/4.0/), which permits unrestricted use, distribution, and reproduction in any medium, provided you give appropriate credit to the original author(s) and the source, provide a link to the Creative Commons license, and indicate if changes were made. The Creative Commons Public Domain Dedication waiver (http://creativecommons.org/publicdomain/zero/1.0/) applies to the data made available in this article, unless otherwise stated. 
due to $S$. aureus. The young patient developed mediumsized arterial infectious vasculitis that led to ischemia and gangrene. We intended to decipher pathogen and host factors that could explain this dramatic presentation.

\section{Case presentation}

\section{Clinical case}

The patient was a 14-year-old male child without notable past medical history and no recent travel. On admission, he had fever, lethargy and diarrhea, and physical examination revealed fever $\left(39^{\circ} \mathrm{C}\right)$, tachycardia, blood pressure $65 / 35 \mathrm{mmHg}$ and poor peripheral perfusion. Several boils were observed on the right elbow. He had subnormal white blood cell count $2.8 \times 10^{9} / \mathrm{L}$ with low lymphocyte count (13\%), a moderate thrombocytopenia and raised CRP (347 mg/L) and procalcitonin plasma levels (279 $\mathrm{ng} /$ $\mathrm{mL}$ ). The diagnosis of septic shock with a possible associated toxic mechanism was retained and intravenous (i.v.) cefotaxime and clindamycin were started with concomitant volume expansion. In the Intensive Care Unit, the patient was intubated as he became confused, needed norepinephrine, inotropic support by epinephrine, and required hemofiltration. Blood cultures showed methicillin susceptible $S$. aureus (MSSA) and treatment was switched to i.v. cloxacillin, clindamycin, and gentamicin. Complementary explorations revealed multiple septic pulmonary abscesses, an abscess of the left occipital lobe, and the absence of endocarditis. In view of septic emboli, clindamycin was switched to fosfomycin in order to have a better cerebral diffusion. The patient then developed ischemia of the four limbs without any purpuric lesion or signs of disseminated intravascular coagulation, which unfortunately rapidly progressed to dry gangrene of the left toes and of the right leg requiring amputation at day 11 after admission. The clinical status of the patient improved slowly. Catecholamines were stopped at day 6, the patient got extubated at day 11 , and he recovered an efficient renal function 5 weeks after his admission. He had no serious neurological injury and a control cerebral MRI was normal three months later.

\section{Histopathology, biological and genetic investigations}

Our initial hypothesis underlying this atypical phenotype was the association of a vasospasm due to high doses of vasopressors and microcirculation disorders due to sepsis. We therefore analyzed the amputated specimen by light microscopy. Samples of left and right amputations were formalin fixed, paraffin embedded, and cut into $3 \mu \mathrm{m}$-thick sections. Sections were stained with Hematoxylin and Eosin, Gram, Periodic Acid Schiff and Grocott staining. The histopathologic analysis revealed perivascular infiltrates of mononuclear cells and neutrophils with arterial involvement (medium-sized vessels) without any capillary lesion (Fig. 1a and b). Furthermore, the arterial endothelium was destroyed and the walls were invaded by Gram-positive cocci with subsequent thrombosis (Fig. 1c and d).

In order to decipher mechanisms underlying these clinical and histopathological findings, pathogen virulence and host factors were analyzed. A wholegenome shotgun library was prepared with Nextera XT Kit (Illumina, San Diego, CA, USA) and sequenced on the MiSeq Illumina sequencing platform $(2 \times 150$ bp paired-end reads). All reads were screened by mapping to known alleles of virulence genes using the Short Read Sequence Typing for Bacterial Pathogens program (SRST2) [8]. Virulence gene allele sequences were retrieved from the virulence factor database (VFDB, http://www.mgc.ac.cn/VFs/). SRST2 was also used to define the sequence type of the strain. Antibiotic-resistance predictions were performed directly from raw reads using "Mykrobe predictor S. aureus" tool [9]. All generated sequences are available at NCBI's BioProject database under accession number PRJNA315766 (http://www.ncbi.nlm.nih. gov/bioproject/PRJNA315766). The sequenced S. aureus isolate was a methicillin-susceptible strain harboring a staphylococcal cassette chromosome (SCC). The patient's strain genome encoded numerous pathogenesis-related genes. The virulence gene equipment of the strain notably included several cytotoxin genes encoding the gamma-hemolysin and the Panton-Valentine (LukSF-PV), LukED and LukGH leukotoxins. It also included genes involved in adhesion and biofilm formation, genes encoding various enterotoxins (A, H, K, and $\mathrm{Q}$ ) and superantigens, exoproteases and immune response evasion proteins (Table 1). The patient's strain belonged to the clonal complex CC1-MSSA-SCCfus [PVL+].

Then, thrombophilic factors and immunity of the patient were examined. Initial low circulating protein antithrombin, $\mathrm{C}$ and $\mathrm{S}$ returned to normal after one month, and other factors associated with hereditary thrombophilia including activated protein $\mathrm{C}$ resistance, lupus anticoagulant and prothrombin G20210A mutation, were absent. Lymphocyte subsets of the patient were determined by routine flow cytometry, serum levels of the IgM, IgA, IgG, and IgG subclasses were assessed by standard nephelometry techniques, and complement was measured via enzyme-linked immunosorbent assay (ELISA). Activation of complement pathways was normal as well as B, T and NK lymphocyte cell count and immunoglobulin levels. Then, activation of cells in whole-blood of the patients were determined after granulocyte isolation by Ficoll density gradient centrifugation. Cells were activated with Toll like receptors agonists, stained with anti-CD62L-FITC, and analyzed by flow cytometry. In vitro stimulation of the patient's peripheral blood mononuclear cells by LPS 

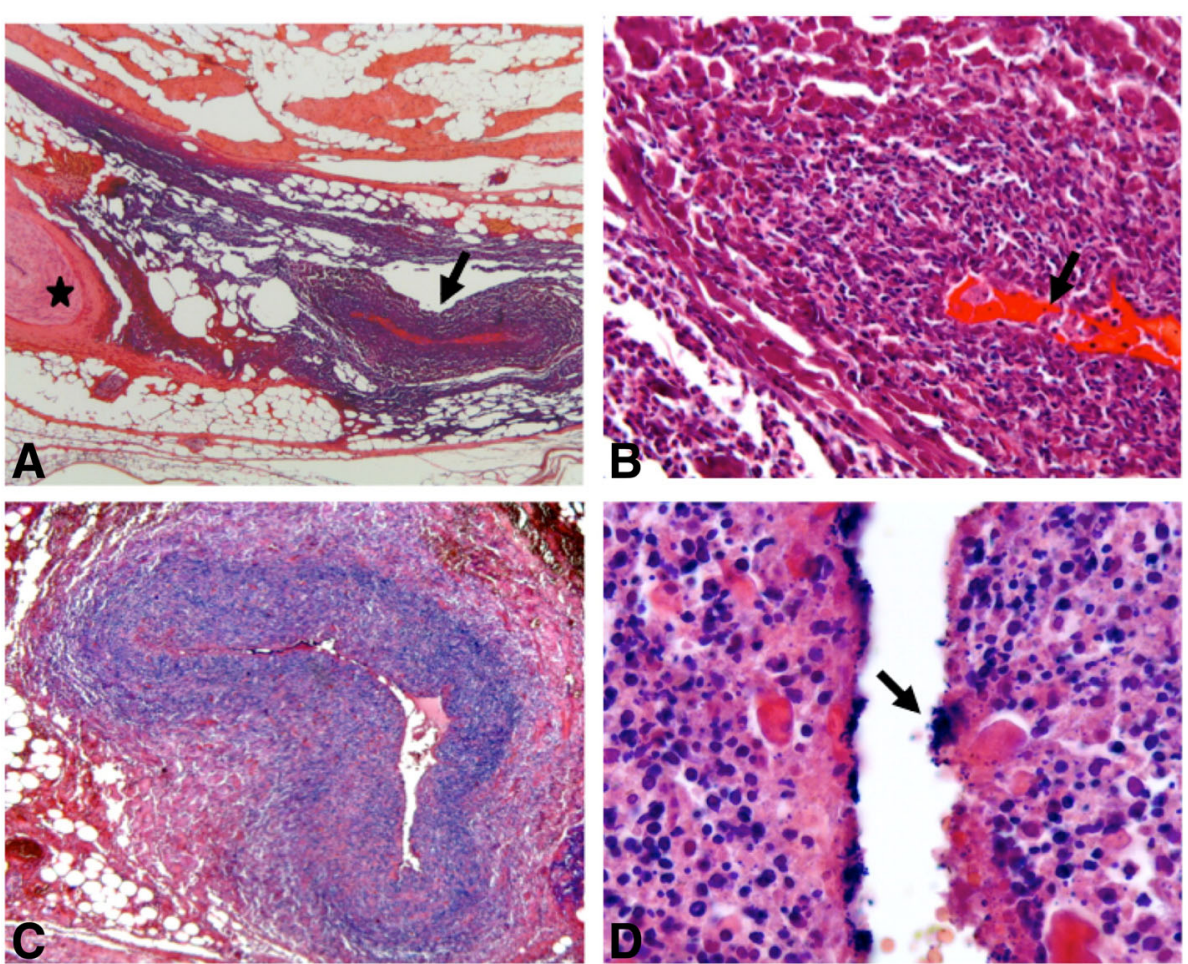

Fig. 1 Histopathology of the amputation specimen. a Coexistence of normal (star) and pathologic medium-sized arteries (arrow) in necrotic and ischemic fibro-adipose tissue. Focal infiltration by inflammatory cells is noted. $\mathbf{b}$ and $\mathbf{c}$ Pathologic artery occluded by fibrinous thrombus (arrow). Arterial wall is replaced by cellular debris without residual endothelium. Surrounding tissues are necrotic and ischemic. $\mathbf{d}$ Gram staining shows bacterial colonization of arterial wall. Numerous bacteria (arrow) are found around the lumen

showed a standard production of cytokines IL-6 and IL-10 by ELISA.

Finally, functional common polymorphisms associated with severe infections and septic shock (PAI-1, rs1799768; MIF, rs755622; ACE rs17326674 IL6, rs1800795; TNF rs1800750, rs1800629, rs361525, and rs909253; TLR2, rs5743708 and rs5743704, TLR4, rs4986790; TLR5, rs5744168; IRAK1, rs1059703; IKB, rs2233406 and rs3138053; FCgammaRIIA, rs1801274 and $\mathrm{CFH}, \mathrm{rs1065489)}[6,7]$ were genotyped using TaqMan Single Nucleotide Polymorphism Genotyping Assays as previously described [7]. Genomic DNA was extracted from mononuclear cells using MagnaPure Compact automate (Roche Diagnostics ${ }^{\circ}$ ). The SNP genotyping revealed that the patient was homozygous for four potential deleterious variants of IRAK1, MIF, ACE, and PAI-I (Table 2).

\section{Discussion and conclusions}

To our knowledge, it is the first reported case of vasculitis occurring during $S$. aureus disseminated infection affecting the medium-sized arteries without any capillary injury. Few cases of extremity necrosis induced by $S$. aureus-producing superantigens have been reported in both adults and children but necrosis was associated with purpura fulminans caused by lesions of the capillaries and subsequent thrombosis [10,11]. Most of these patients had a fatal outcome due to septic shock or displayed gangrene of the distal extremities. This uncommon phenotype could be the consequence of the interaction of a virulent pathogen and a susceptible host.

Recently, an emergence of CC1-MSSA has been reported [12, 13]. However, to our knowledge, no severe cases due to CC1-MSSA clone had been reported so far. The PVL toxin and the four other enterotoxins isolated from our patient are likely to be responsible for a massive cytokine release with subsequent toxic shock syndrome and transient alteration of the immune system [14]. Staphylococcal superantigens could also be responsible for an inflammatory vessel vasculitis. However, the association between superantigen production and poor prognosis of MSSA infection is still under debate [15]. $S$. aureus is also known to interact with the endovascular system through the expression of numerous adhesins leading to specific infection such as infective endocarditis, suppurative thrombophlebitis, or vascular graft infection [4]. ClfB, cna and sea genes that encode respectively clumping factor B, collagen adhesin and enterotoxin A, were present in the patient strain. However, the venular endothelium seems to be the predominant target for $S$. 
Table 1 Significant virulence genes identified by wholegenome sequencing of the clinical isolate

\begin{tabular}{ll}
\hline Gene name & Description \\
\hline Adhesion factors & \\
cna & Collagen-binding adhesin precursor \\
ebpS & Elastin-binding protein \\
IffA, clfB & Clumping factors \\
fnbA, fnbB & Fibronectin-binding proteins \\
sasG & Staphylococcus aureus surface protein G \\
sdrC, sdrD, sdrE & Serine-aspartate repeat proteins \\
Biofilm-associated genes \\
icaADB & intercellular adhesion proteins \\
icaR & ica operon transcriptional regulator \\
Cytotoxins & \\
hlgABC & gamma-hemolysin \\
lukED & leukotoxin ED \\
lukGH & leukotoxin GH \\
lukSF-PV & Panton-Valentine leukocidin \\
Enterotoxins & \\
sea, seh & staphylokinase precursor \\
sek, sea & staphopain B cysteine protease \\
Staphylococcal superantigen-like \\
set(s) & superantigen-like proteins \\
Proteases & \\
splABCDEF & senterotoxin-like proteins \\
sspA &
\end{tabular}

aureus binding. We only provided here a list of virulence genes present in the strain, but variations in the expression of these genes or in protein production could be responsible for the dramatic clinical presentation and the extreme prothrombotic phenotype observed in our patient [16].

Associated with virulent factors, our data argue for direct role of unusual immune and coagulation system responses of the host. The expression of adhesion molecules on endothelial cell wall is up-regulated during sepsis and might help colonization by the pathogen [4]. A hyperinflammatory state associated with dysfunction of the endothelium in sepsis can lead to the disturbance of the coagulation balance and subsequent thrombosis $[2,17]$. This may be facilitated by the presence of functional variants of genes involved in inflammatory response [6]. Indeed, our patient was homozygous for 4 polymorphisms associated with higher susceptibility and/or severity of
Table 2 Genotype findings of the patient at all loci tested. Homozygous potential deleterious variants were observed for four genes: IRAKI, MIF, ACE involved in inflammation and PAI-1 in thrombotic events

\begin{tabular}{|c|c|c|c|c|}
\hline Gene name & Ref & WTMT & WT/M & $\mathrm{M} / \mathrm{M}$ \\
\hline PAl-1 & rs1799768 & & & $x$ \\
\hline MIF & rs755622 & & & $x$ \\
\hline$A C E$ & rs17326674 & & & $x$ \\
\hline IL-6 & rs1800795 & $x$ & & \\
\hline \multirow[t]{4}{*}{ TNF } & rs1800750 & $x$ & & \\
\hline & rs1800629 & $x$ & & \\
\hline & rs361525 & $x$ & & \\
\hline & rs909253 & $x$ & & \\
\hline$T L R 2$ & rs5743708 & $x$ & & \\
\hline$T L R 2$ & rs5743704 & $x$ & & \\
\hline TLR4 & rs4986790 & $x$ & & \\
\hline TLR5 & rs5744168 & $x$ & & \\
\hline IRAK1 & rs1059703 & & & $x$ \\
\hline \multirow[t]{2}{*}{$I K B$} & rs2233406 & $x$ & & \\
\hline & rs3138053 & $x$ & & \\
\hline FcgammaRIIA & rs1801274 & $x$ & & \\
\hline $\mathrm{CFH}$ & rs1065489 & $x$ & & \\
\hline
\end{tabular}

PAl-1 Plasminogen activator inhibitor 1, MIF Macrophage migration inhibitor factor, ACE Angiotensin converting enzyme, IL-6 Interleukin 6, TNF Tumor Necrosis Factor, TLR Toll like receptor, IRAK1 Interleukin-1 receptor associated kinase, IKB Inhibitory protein of nuclear factor-KB, FCgammaRIIA Fc receptor for IgG, CFH Complement factor $\mathrm{H}, W T$ Wild type concerns the frequent allele, $M$ Mutation concerns the variant rare allele, Ref Reference number for the studied SNP

severe sepsis: IRAK1, ACE, PAI-1 and MIF genes [6, 7, 18, 19]. In particular, $P A I-1$ (gene encoding for plasminogen activator inhibitor-1) variant is associated with decreased fibrinolysis and a higher risk of amputation of the extremities in septic shock $[6,18]$. Even if the final effect of combination of these four potentially deleterious polymorphisms is unknown, it might explain in part the increased inflammatory and pro-coagulant state, and the severity of the staphylococcal infection in our patient.

In conclusion, disseminated $S$. aureus infection, apart from septic shock, could lead to arterial vasculitis and arterial thrombosis, with severe consequences such as limb amputation. The combination of pathogen virulence and genetic variability of the host response probably explain the dramatic severity of this infection. The investigation of predisposing factors might help for future tailor-made adjunctive therapy in sepsis.

\section{Abbreviations}

ACE: Angiotensin l-converting enzyme; CRP: C-reactive protein;

ELISA: Enzyme-liked immunosorbent assay; IRAK: Interleukin-1 receptorassociated kinase 1; MIF: Macrophage inhibition inhibitory factor; MRI: Magnetic resonance imaging; PAI-I: Plasminogen activator inhibitor type I; PVL: Panton-Valentine leukocidin; SCC: Staphylococcal cassette chromosome; SNP: Single polymorphism nucleotid 


\section{Acknowledgements}

None.

\section{Funding}

There is no financial support for the study to declare.

\section{Availability of data and materials}

Most of the data generated and/or analyzed are available in the current study. If necessary, all genomic data could be available from the corresponding author on reasonable request.

\section{Authors' contributions}

CV and JT wrote the report. CV, FM, OL and JT managed the patients. LG interpreted histopathologic slides. CV and LG created the figures. XN, AJ, DB, CP, AC and JPM carried out laboratory testing and interpreted the data. All authors were involved in the writing and/or revision of the manuscript. All authors have read and approved the manuscript.

\section{Ethics approval}

Not applicable.

\section{Consent for publication}

Written consents from the patient and his parents were obtained for publication of this case report, images and all information contained in it.

\section{Competing interests}

The authors declare that they have no competing interests.

\section{Publisher's Note}

Springer Nature remains neutral with regard to jurisdictional claims in published maps and institutional affiliations.

\section{Author details}

${ }^{1}$ Department of Microbiology, Necker Enfants-malades hospital, APHP, Paris Descartes University, Paris, EU, France. ${ }^{2}$ Department of Pediatric Intensive Care Unit, Necker Enfants-Malades Hospital, APHP, Paris Descartes University, Paris, EU, France. ${ }^{3}$ Pathology Department, Necker Enfants-Malades Hospital, APHP, Paris Descartes University, Paris, EU, France. ${ }^{4}$ Department of Hematology, Necker Enfants-Malades Hospital, APHP, Paris Descartes University, Paris, EU, France. ${ }^{5}$ Necker-Enfants-Malades Institute, INSERM U1151; CNRS UMR8253, Paris, France. ${ }^{6}$ Center for the Study of Primary Immunodeficiencies, Necker Enfants Malades Hospital, APHP, Paris Descartes University, Paris, EU, France. ${ }^{7} \mathrm{HU}$ Imagine, Laboratory of Human Genetics of Infectious Diseases, INSERM U1163, Paris, EU, France. ${ }^{8}$ Medical Intensive Care Unit, Cochin Hospital, AP-HP, Paris Descartes University, Paris, EU, France. ${ }^{9}$ Department of Infection, Immunity and Inflammation, Institut Cochin, INSERM U1016, Paris, EU, France. ${ }^{10}$ Department of Infectious Diseases and Tropical Medicine, Necker Enfants-Malades Hospital, Necker-Pasteur Infectious Diseases Center, Université Paris Descartes, IHU Imagine, Paris, EU, France. ${ }^{11}$ Department of General Pediatrics and Pediatric Infectious Diseases, Necker Enfants-Malades Hospital, APHP, Paris Descartes University, 149 rue de Sèvres, 75015 Paris, EU, France.

Received: 4 November 2018 Accepted: 24 March 2019

Published online: 03 April 2019

\section{References}

1. Lowy FD. Staphylococcus aureus infections. N Engl J Med. 1998;339(8):520-32.

2. Powers ME, Bubeck Wardenburg J. Igniting the fire: Staphylococcus aureus virulence factors in the pathogenesis of sepsis. PLoS Pathog. 2014;10(2): e1003871.

3. Yoong P, Torres VJ. The effects of Staphylococcus aureus leukotoxins on the host: cell lysis and beyond. Curr Opin Microbiol. 2013;16(1):63-9.

4. Chavakis T, Wiechmann K, Preissner KT, Herrmann M. Staphylococcus aureus interactions with the endothelium: the role of bacterial "secretable expanded repertoire adhesive molecules" (SERAM) in disturbing host defense systems. Thromb Haemost. 2005;94(2):278-85.

5. Casanova JL. Severe infectious diseases of childhood as monogenic inborn errors of immunity. Proc Natl Acad Sci U S A. 2015;112(51):E7128-37.

6. Arcaroli J, Fessler MB, Abraham E. Genetic polymorphisms and sepsis. Shock 2005;24(4):300-12
7. Toubiana J, Courtine E, Pene F, Viallon V, Asfar P, Daubin C, et al. IRAK1 functional genetic variant affects severity of septic shock. Crit Care Med. 2010;38(12):2287-94

8. Inouye M, Dashnow H, Raven LA, Schultz MB, Pope BJ, Tomita T, et al. SRST2: rapid genomic surveillance for public health and hospital microbiology labs. Genome Med. 2014;6(11):90.

9. Bradley P, Gordon NC, Walker TM, Dunn L, Heys S, Huang B, et al. Rapid antibiotic-resistance predictions from genome sequence data for Staphylococcus aureus and mycobacterium tuberculosis. Nat Commun. 2015;6:10063.

10. Fitzgerald CJ, Pranikoff TV, Ross GA, Mou S, Givner LB, Shetty AK. Purpura fulminans caused by community-associated methicillin-resistant staphylococcus aureus. Am J Emerg Med. 2012;30(6):1013 e1011-4.

11. Kravitz GR, Dries DJ, Peterson ML, Schlievert PM. Purpura fulminans due to Staphylococcus aureus. Clin Infect Dis. 2005:40(7):941-7.

12. Baines SL, Howden BP, Heffernan H, Stinear TP, Carter GP, Seemann T, et al. Rapid emergence and evolution of Staphylococcus aureus clones harboring fusC-containing staphylococcal cassette chromosome elements. Antimicrob Agents Chemother. 2016;60(4):2359-65.

13. Bourigault C, Corvec S, Brulet V, Robert PY, Mounoury O, Goubin C, et al. Outbreak of skin infections due to Panton-valentine Leukocidin-positive methicillin-susceptible Staphylococcus aureus in a French prison in 20102011. PLoS Curr. 2014;6.

14. Spaulding AR, Salgado-Pabon W, Kohler PL, Horswill AR, Leung DY, Schlievert PM. Staphylococcal and streptococcal superantigen exotoxins. Clin Microbiol Rev. 2013:26(3):422-47.

15. van Timmeren MM, Heeringa $P$, Kallenberg CG. Infectious triggers for vasculitis. Curr Opin Rheumatol. 2014:26(4):416-23.

16. Jenkins A, Diep BA, Mai TT, Vo NH, Warrener P, Suzich J, et al. Differential expression and roles of Staphylococcus aureus virulence determinants during colonization and disease. MBio. 2015;6(1):e02272-14.

17. Angus DC, van der Poll T. Severe sepsis and septic shock. N Engl J Med. 2013:369(21):2063.

18. Garcia-Segarra G, Espinosa G, Tassies D, Oriola J, Aibar J, Bove A, et al. Increased mortality in septic shock with the $4 \mathrm{G} / 4 \mathrm{G}$ genotype of plasminogen activator inhibitor 1 in patients of white descent. Intensive Care Med. 2007:33(8):1354-62.

19. Savva A, Brouwer MC, Roger T, Valls Seron M, Le Roy D, Ferwerda B, et al. Functional polymorphisms of macrophage migration inhibitory factor as predictors of morbidity and mortality of pneumococcal meningitis. Proc Natl Acad Sci U S A. 2016:113(13):3597-602.

Ready to submit your research? Choose BMC and benefit from

- fast, convenient online submission

- thorough peer review by experienced researchers in your field

- rapid publication on acceptance

- support for research data, including large and complex data types

- gold Open Access which fosters wider collaboration and increased citations

- maximum visibility for your research: over $100 \mathrm{M}$ website views per year

At BMC, research is always in progress.

Learn more biomedcentral.com/submissions 